ARTICLE

DOI: $10.1038 / s 41467-017-00693-3$

\title{
Tango7 regulates cortical activity of caspases during reaper-triggered changes in tissue elasticity
}

Yunsik Kang ${ }^{1,2,4}$, Sarah D. Neuman (10 ${ }^{1,3}$ \& Arash Bashirullah ${ }^{1,2,3}$

Caspases perform critical functions in both living and dying cells; however, how caspases perform physiological functions without killing the cell remains unclear. Here we identify a novel physiological function of caspases at the cortex of Drosophila salivary glands. In living glands, activation of the initiator caspase dronc triggers cortical F-actin dismantling, enabling the glands to stretch as they accumulate secreted products in the lumen. We demonstrate that tango7, not the canonical Apaf-1-adaptor dark, regulates dronc activity at the cortex; in contrast, dark is required for cytoplasmic activity of dronc during salivary gland death. Therefore, tango7 and dark define distinct subcellular domains of caspase activity. Furthermore, tango7-dependent cortical dronc activity is initiated by a sublethal pulse of the inhibitor of apoptosis protein (IAP) antagonist reaper. Our results support a model in which biological outcomes of caspase activation are regulated by differential amplification of IAP antagonists, unique caspase adaptor proteins, and mutually exclusive subcellular domains of caspase activity.

\footnotetext{
${ }^{1}$ Division of Pharmaceutical Sciences, University of Wisconsin-Madison, 777 Highland Avenue, Madison, WI 53705-2222, USA. ${ }^{2}$ Laboratory of Genetics Graduate Program, University of Wisconsin-Madison, Madison, WI 53705, USA. ${ }^{3}$ Cellular and Molecular Biology Graduate Program, University of Wisconsin-Madison, Madison, WI 53705, USA. ${ }^{4}$ Present address: Vollum Institute, Oregon Health \& Sciences University, Portland, OR 97239-3098, USA. Correspondence and requests for materials should be addressed to A.B. (email: bashirullah@wisc.edu)
} 
C aspases are cysteine-aspartic proteases best known for their roles in initiating cellular demolition during apoptosis $^{1,2}$. These proteins are maintained within the cell as inactive zymogens. However, once activated, a caspase cascade is released, resulting in critical consequences for the cell. Initiator caspases sit at the top of this cascade. These proteins exist in the cell as inactive monomers, but upon receipt of an activating stimulus, they form active dimers, mediated by adaptor proteins ${ }^{3}$. The best characterized activation platform, which is required during apoptosis, is the apoptosome ${ }^{4,5}$. The apoptosome consists of the initiator caspase, caspase- 9 (dronc in Drosophila), and the adaptor protein Apaf-1 (dark in Drosophila). In mammals, release of cytochrome $c$ activates the apoptosome, allowing caspase- 9 to cleave a second class of caspases, the effector caspases. The effector caspases exist in the cell as inactive dimers, and require cleavage by initiator caspases for activation ${ }^{6,7}$. The major effector caspases in Drosophila are drice and $d c p-1$. Activated effector caspases then cleave critical cellular targets as well as other effector caspases, generating a cascade of caspase activity that eventually results in cellular demolition. This self-perpetuating cascade of caspase activation led to the widespread notion that caspase activation represents a "point of no return" in the life of a cell. However, over the last decade, there have been many examples of caspases playing non-lethal, or "non-apoptotic," roles in cells that do not die.

The list of non-lethal, non-apoptotic roles of caspases has been steadily growing ${ }^{8-13}$. For example, the initiator caspase, caspase-9, and the effector caspase, caspase-3, have been shown to mediate axon pruning during local deprivation of $\mathrm{NGF}^{14}$ Additionally, mouse hair follicles and the surrounding cells require non-apoptotic functions of the effector caspase, caspase-7, for proper development ${ }^{15}$. In Drosophila, the initiator caspase dronc plays a critical role in dendrite pruning of the sensory neurons of the peripheral nervous system ${ }^{16,17}$, and also plays an important role in sperm individualization ${ }^{18-20}$. Although many non-apoptotic functions of caspases have been identified, how caspases function without executing the cell has remained a mystery. Unfortunately, these lethal and non-lethal outcomes of caspase activation have been studied in different cell types, making mechanistic comparisons very difficult.

We have found that the Drosophila larval salivary glands provide an ideal model to study developmentally regulated non-lethal and lethal functions of caspases in a single cell type. Here we examine two distinct caspase activation events during salivary gland development: one resulting in a non-apoptotic, non-lethal outcome and the second resulting in a lethal outcome.

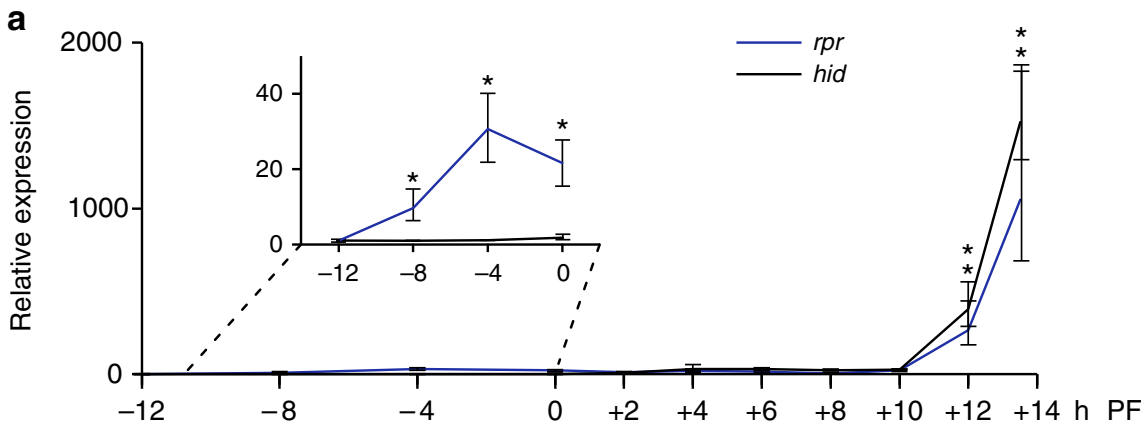

b

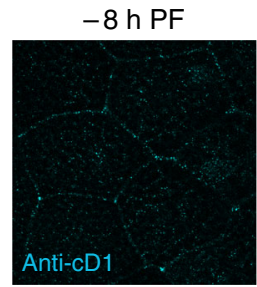

$-4 \mathrm{~h}$ PF

$-1 \mathrm{~h}$ PF
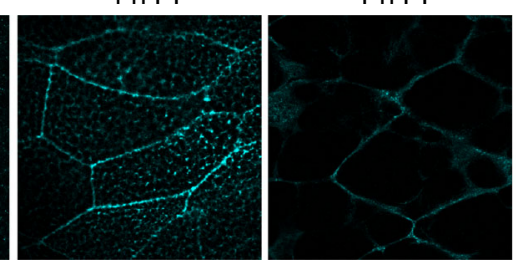

O h PF

C

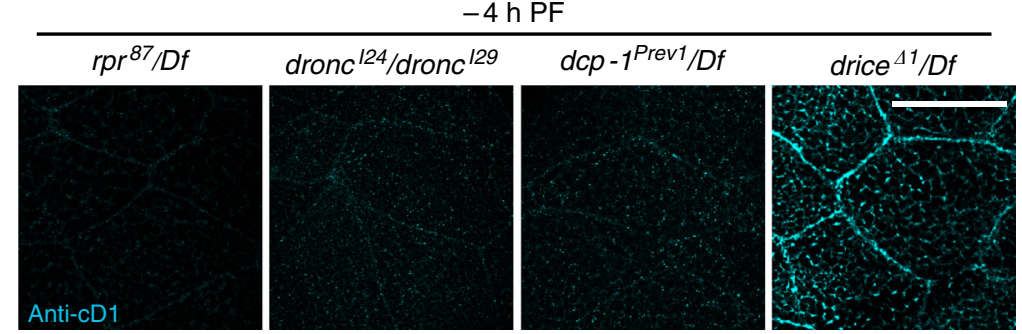

Fig. 1 A low amplitude pulse of reaper (rpr) triggers caspase activation at the cortex of salivary glands. a qPCR analysis of rpr (blue line) and head involution defective (hid) (black line) expression in salivary glands at the onset of metamorphosis. Both rpr and hid are induced $>1000$-fold at the start of pupal development, while only rpr is induced ( 30-fold) at the end of larval development. $y$-axis shows relative expression; $x$-axis shows developmental stage in hours relative to the onset of metamorphosis. Expression levels shown relative to the lowest point for each gene and normalized to the reference gene rp49. Three biological samples analyzed for each stage; error bars represent standard error determined by REST analysis (see "Methods"); asterisks indicate $p$-value $<0.05$ calculated by REST analysis. b Timecourse of cleaved-Dcp1 (anti-cD1) antibody staining in salivary glands at the end of larval development. Anti-cD1, in cyan, does not exhibit specific staining at $-8 \mathrm{~h}$ PF, but stains strongly at the cortex at $-4 \mathrm{~h}$ PF. Cortical staining of cD1 persists through $-1 \mathrm{~h}$ PF, but diminishes at $\mathrm{O}$ PF. c Regulation of anti-cD1 cortical staining in salivary glands at $-4 \mathrm{~h}$ PF. Cortical cD1 staining is absent in rpr, dronc, and dcp-1 mutant salivary glands, but present in drice mutant salivary glands. Scale bars represent $100 \mu \mathrm{m}$. PF, puparium formation, Df, deficiency 
a

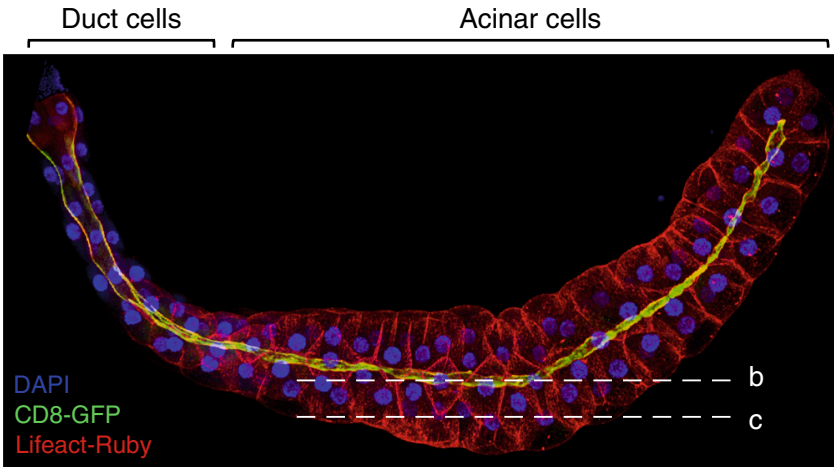

d
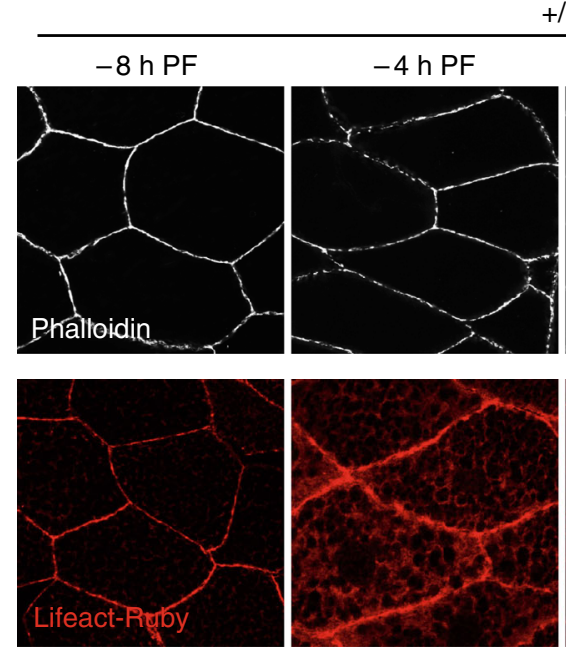

f

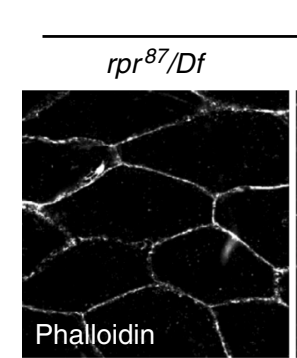

g

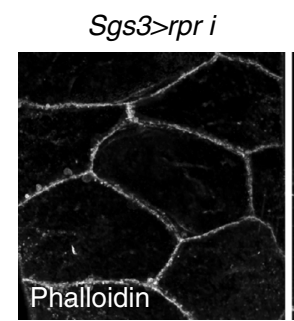

$+/+$

\begin{abstract}
$-1 \mathrm{~h}$ PF
\end{abstract}
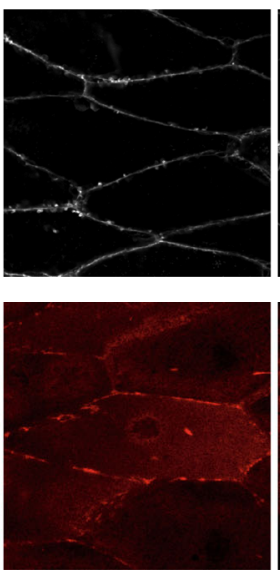

0 h PF $d c p-1^{\text {Prev1}} / D f$

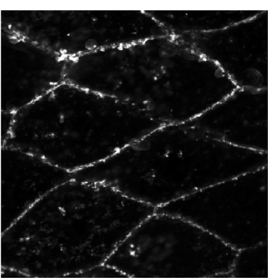

Sgs3>diap 1
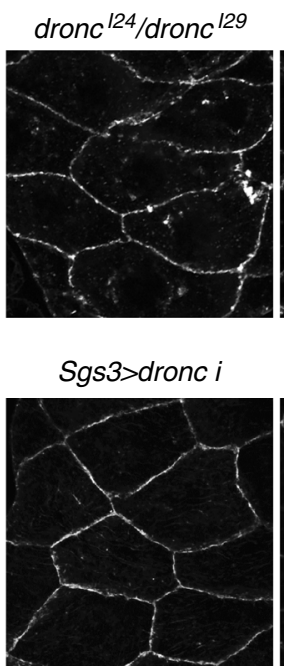

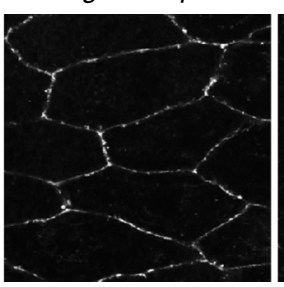

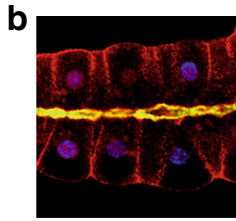

C

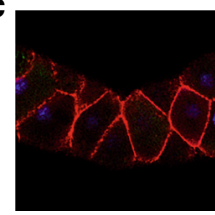

$0 \mathrm{hPF}$
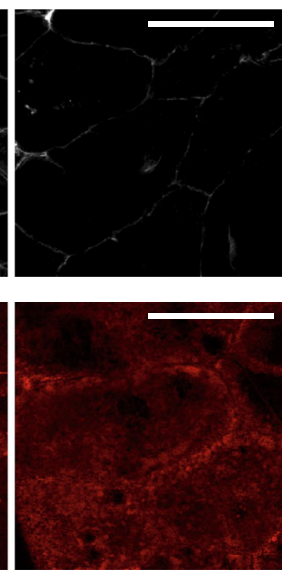
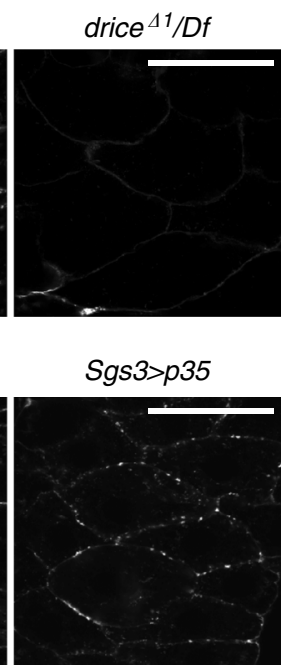

Fig. 2 A rpr-triggered caspase cascade dismantles the cortical F-actin cytoskeleton in living salivary glands. a Maximal intensity projection of a whole salivary gland at $-8 \mathrm{~h}$ PF expressing the actin-binding peptide Lifeact-Ruby in red and the membrane marker CD8-GFP in green, both under control of the salivary gland driver fkh-GAL4. CD8-GFP is enriched at the apical membrane (proximity of Lifeact-Ruby and CD8-GFP at the apical membrane appears yellow); nuclei stained by DAPI in blue. b, c Confocal slices through the salivary glands. b A medial slice shows an apical-basal view of acinar cells, with the lumen in the middle. c A lateral slice shows a "coronal" view, highlighting the cortical F-actin cytoskeleton. d, e Cortical F-actin breaks down at the end of larval development. d Phalloidin staining (white) in salivary glands at $-8 \mathrm{~h}$ PF reveals tight cortical bundles of F-actin; this structure begins to disintegrate at $-4 \mathrm{~h} \mathrm{PF}$ and $-1 \mathrm{~h} \mathrm{PF}$, with no phalloidin staining visible by $\mathrm{O}$ PF. e Lifeact-Ruby in salivary glands confirms cortical F-actin breakdown. Lifeact-Ruby reveals that most of the actin in the cell is present at the cortex at $-8 \mathrm{~h}$ PF. Breakdown of F-actin is reflected by a gradual redistribution of Lifeact-Ruby signal from the cortex to the cytoplasm at $-4 \mathrm{~h} \mathrm{PF}$ and $-1 \mathrm{~h} P F$, with only cytoplasmic signal visible at $\mathrm{O}$ PF. $\mathbf{f}$ F-actin dismantling requires caspase activity. F-actin breakdown is blocked in rpr, dronc, and dcp-1 mutant salivary glands, but not in drice mutant salivary glands. $\mathbf{g}$ Caspases regulate F-actin breakdown cellautonomously. Salivary gland-specific (using Sgs3-GAL4) overexpression of rpr-RNAi, dronc-RNAi, or of the caspase inhibitors diap1 or p35 inhibits F-actin breakdown. Scale bars represent $100 \mu \mathrm{m}$. Df, deficiency, PF, puparium formation 
We find that these two events are both regulated by the steroid hormone ecdysone; however, differential signaling mechanisms selectively amplify the activating signal, IAP antagonist expression, to generate a lethal outcome instead of a non-lethal response. Moreover, we also demonstrate that caspases can be activated in mutually exclusive subcellular domains to accomplish different biological functions, and the use of different adaptor proteins mediates this mutually exclusive activation. Finally, our results highlight a novel, non-lethal function for caspases in the control tissue elasticity during exocrine secretion events. Altogether, we provide a new model for how caspases can be activated and perform cellular functions without triggering cell death during development.

\section{Results}

A regulated sublethal pulse of $r p r$ in salivary glands. In Drosophila, caspase activation hinges on transcriptional induction of IAP antagonists ${ }^{21}$, proteins that remove inhibitor of apoptosis proteins (IAPs) and initiate a caspase cascade. The primary IAP antagonists in Drosophila are reaper $(r p r)$ and head involution defective ( $h i d$ ), and these proteins play a critical role in the programmed cell death of the larval salivary glands during metamorphosis $^{22}, 23$. Our gene expression studies in the larval salivary glands at the onset of metamorphosis revealed a 1000 -fold induction of hid at the start of pupal development (Fig. 1a). In contrast, we observed two distinct pulses of $r p r$ expression: a 30-fold induction at the end of larval development, and a 1000-fold induction at the start of pupal development (Fig. 1a). The late, large pulse of $r p r$ and hid has previously been characterized as part of the larval salivary gland cell death response; 22,23 however, the early, small pulse of $r p r$ has not been described before. We wanted to confirm that this small $r p r$ pulse was biologically relevant, so we first tested if the pulse was developmentally regulated. The large, lethal pulse of IAP antagonists is induced by the prepupal pulse of the steroid hormone 20-hydroxyecdysone (henceforth called ecdysone $)^{23}$. Another ecdysone pulse occurs at the end of larval development $^{24}$, and peak steroid hormone levels coincide with the timing of the small pulse of rpr expression. We therefore tested if this small $r p r$ pulse was regulated by ecdysone signaling. We found that tissue-specific expression of a dominant negative form of the ecdysone receptor $\left(E c R^{F 645 A}\right)$ abolished $r p r$ expression at the end of larval development (Supplementary Fig. 1a), indicating that this small $r p r$ pulse is developmentally regulated by the late larval pulse of ecdysone.

Although ecdysone signaling initiates induction of both the small and large $r p r$ pulses, the mechanisms mediating the difference in magnitude between these pulses were unclear. We tested if this expression difference was regulated by different downstream targets of ecdysone. Several transcription factors, including $B R-C, E 74 A$, and $M e d 24$, are required for salivary gland cell death, and these transcription factors are themselves induced by ecdysone $23,25,26$. We found that BR-C, E74A, and Med24 mutant salivary glands had reduced expression of $r p r$ at the late, lethal pulse (Supplementary Fig. 1b). In $B R-C$ mutant salivary glands $\left(r b p^{5}\right)$, this resulted in $r p r$ expression levels that resembled the magnitude of the early, small larval pulse. Interestingly, these same three mutants did not affect $r p r$ expression at the small, early pulse (Supplementary Fig. 1a). Taken together, these results indicate that downstream targets of ecdysone, like BR-C, E74A, and Med24, differentially amplify rpr expression during the death response, making it possible to generate both sublethal and lethal pulses of the IAP antagonist. Furthermore, the early, sublethal pulse of rpr was induced in a tissue-specific manner. Although all tissues tested responded to ecdysone by inducing expression of the primary target gene $E 74 A$, only the salivary glands and midgut induced rpr expression, while the wing discs and central nervous system did not (Supplementary Fig. 2). These results suggest that lethal vs. sublethal pulses of $r p r$ are developmentally controlled, raising the intriguing possibility that differential amplification and tissue-specific expression of IAP antagonists may play a role in determining apoptotic vs. non-apoptotic outcomes of caspase activation.

Sublethal rpr pulse initiates cortical caspase activation. To determine if the sublethal pulse of $r p r$ in salivary glands had a function, we first examined if caspases were activated at this stage. We have previously shown that staining glands at this stage with antibodies directed to cleaved caspase-3 (anti-cC3) does not show any signs of caspase activation ${ }^{27}$. Staining with antibodies directed to cleaved-Dcp-1 (anti-cD1), however, showed staining primarily at the cell cortex, with the intensity of staining peaking at $-4 \mathrm{~h}$ PF ( $4 \mathrm{~h}$ before puparium formation) (Fig. 1b). This Dcp-1 activation profile coincides with the peak of $r p r$ expression, suggesting that the two events may be related. Indeed, in $r p r$ mutant glands, anti-cD1 staining was disrupted (Fig. 1c). Furthermore, anti-cD1 staining required the initiator caspase dronc and the effector caspase $d c p-1$, but not the effector caspase drice (Fig. 1c). Importantly, these results indicate that the small, sublethal pulse of $r p r$ initiates activation of specific caspases at the cortex of developing larval salivary glands.

Caspases dismantle cortical F-actin in living glands. To identify the consequence of caspase activation at the cortex of the larval salivary glands, we tested several vital dyes and antibodies known to stain the cell cortex. Only phalloidin, a vital dye that binds to filamentous actin (F-actin), showed a dramatic difference in staining between $-8 \mathrm{~h} \mathrm{PF}$ and $0 \mathrm{~h}$ PF glands. The cortex of most living cells features a meshwork of F-actin bundles that confers shape and structural stability to the cell. Accordingly, we observed a meshwork of cortical F-actin framing every cell in the larval salivary glands (Fig. 2a). The acinar cells of the salivary gland form a radially symmetric polarized epithelium; the apical membrane, visualized by expression of a membrane-targeted GFP (CD8-GFP) (Fig. 2a, b), outlines the lumen of the glands, while the basal membrane faces the exterior of the gland. A coronal view of the salivary glands revealed a clear F-actin cytoskeleton outlining each cell (Fig. 2c). However, we found that this cortical F-actin cytoskeleton was dismantled at the end of larval development. At $-8 \mathrm{~h} \mathrm{PF}$, phalloidin staining showed tight bundles of cortical F-actin (Fig. 2d). Lifeact-Ruby, a fluorescently tagged peptide that binds actin ${ }^{28,29}$, also showed that most of the actin within the cell at $-8 \mathrm{~h} \mathrm{PF}$ is present at the cortex (Fig. 2e). These phalloidin-stained F-actin bundles became less distinct at -4 and $-1 \mathrm{~h} \mathrm{PF}$, and disappeared by PF (Fig. 2d). Consistently, Lifeact-Ruby imaging showed that this loss of cortical F-actin was associated with a gradual redistribution of actin from the cortex to the cytoplasm (Fig. 2e). This dissolution of F-actin appeared to start at $-4 \mathrm{~h} \mathrm{PF}$, when the decrease in phalloidin staining was accompanied by increased Lifeact-Ruby signal at the cortex, as well as an increase in Lifeact-Ruby signal in the cytoplasm (cf. Lifeact-Ruby vs. phalloidin staining; Fig. 2d, e). These results reflect a rapid disassembly of cortical actin filaments into cytoplasmic monomers beginning at $-4 \mathrm{~h} \mathrm{PF}$, at the peak of cortical caspase activation.

We next tested if cortical F-actin breakdown depended on the sublethal pulse of $r p r$ and subsequent caspase activation at this stage. Indeed, loss of $r p r$ function blocked dismantling of the F-actin cytoskeleton (Fig. 2f). Moreover, F-actin disassembly required dronc and $d c p-1$ but not drice (Fig. 2f), mirroring 


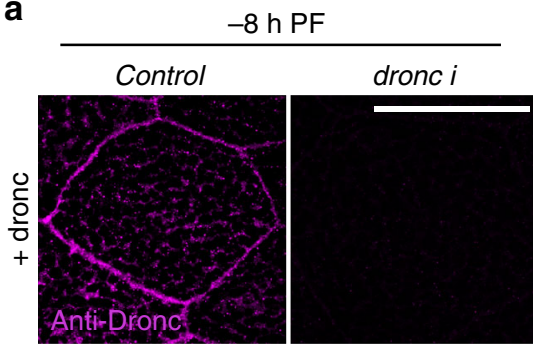

C
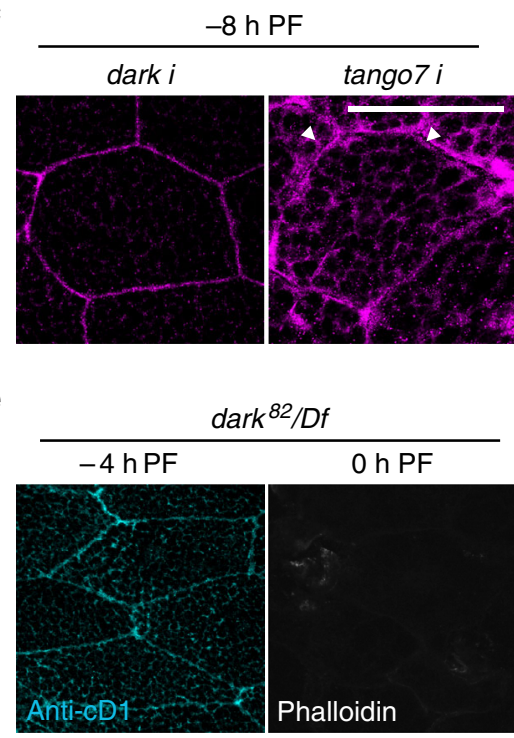

b

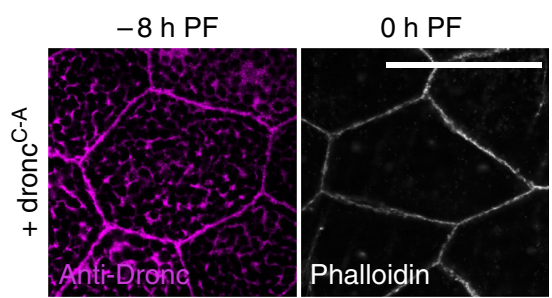

d

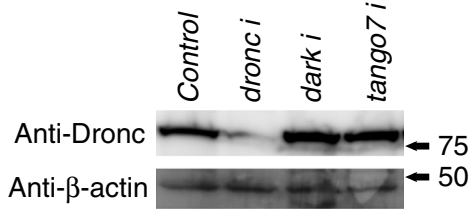

f

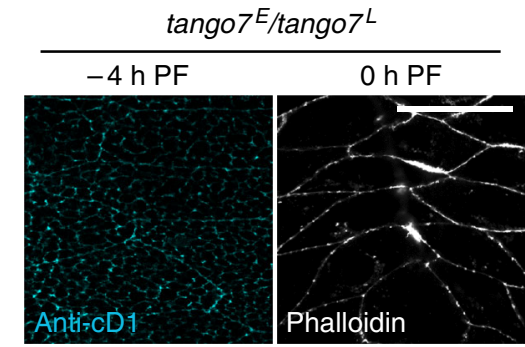

Fig. 3 Tango7 is required for cortical localization and activity of Dronc in living salivary glands. a Dronc localizes to the cortex of salivary glands at the end of larval development. Immunofluorescent staining using an antibody to detect Dronc protein (anti-Dronc, in magenta) in salivary glands overexpressing dronc under control of Sgs3-GAL4 ( + dronc) shows that Dronc localizes to the cortex of salivary gland cells at - $8 \mathrm{~h}$ PF. Co-expression of dronc-RNAi abolishes anti-Dronc staining. b F-actin dismantling requires Dronc catalytic activity. Anti-Dronc staining in salivary glands overexpressing a catalytically inactive Dronc ( + dronc $^{C-A}$, in magenta) under control of Sgs3-GAL4 reveals that Dronc ${ }^{C-A}$ still localizes to the cortex at $-8 \mathrm{~h}$ PF. However, phalloidin staining, in white, shows that F-actin does not break down in these glands at 0 h PF. c tango7, not dark, regulates cortical localization of Dronc. Anti-Dronc staining, in magenta, in salivary glands overexpressing dronc under control of Sgs3-GAL4 shows that Dronc still localizes to the cortex upon co-expression of RNAi against dark. In contrast, tango7 RNAi knockdown causes Dronc to diffuse away from the cortex toward the cytoplasm, with clear gaps lacking anti-Dronc staining visible at the cortex (white arrowheads). The cytoplasm at this stage is filled with secretory granules (black "spots"). d Dronc levels are not reduced in tango7-RNAi salivary glands. Western blot analysis of Dronc protein expression levels in dronc-RNAi, dark-RNAi, and tango7-RNAi salivary glands (under control of Sgs3-GAL4) shows that Dronc levels are dramatically reduced upon overexpression of dronc-RNAi, but are not decreased upon expression of dark-RNAi or tango7-RNAi. Salivary glands were dissected at $-8 \mathrm{~h}$ PF; $\beta$-actin used as loading control. e, $\mathbf{f}$ tango7, not dark, is required for cortical caspase activation and dismantling of F-actin. e Cortical anti-cD1 staining, in cyan, is not disrupted in dark mutant salivary glands at -4 h PF. Phalloidin staining, in white, shows that F-actin breaks down normally in dark mutant salivary glands. $\mathbf{f}$ In contrast, cortical anti-cD1 staining is abolished at - $4 \mathrm{~h}$ PF and F-actin fails to break down at $\mathrm{O}$ PF in tango7 mutant salivary glands. Scale bars represent $100 \mu \mathrm{m}$. Df, deficiency, PF, puparium formation

the caspase cascade required for cortical staining of anti-cD1 during this stage (Fig. 1c). To further validate the role of caspases in F-actin disassembly, we tested the effect of inhibitors of caspase activation. Salivary gland-specific expression of diap1, the primary Drosophila IAP, disrupted F-actin breakdown, while expression of $p 35$, the Baculovirus-derived effector caspase inhibitor, slightly inhibited this process (Fig. 2g). Importantly, salivary gland-specific RNAi knockdown of rpr or dronc strongly inhibited F-actin disassembly (Fig. 2g). These results indicate a cell-autonomous requirement for rpr-dependent antagonism of diapl and activation of dronc during F-actin breakdown in the larval salivary glands.

Given that the small pulse of $r p r$ is dependent on ecdysone signaling, we also tested if ecdysone directly triggered the loss of cortical F-actin in salivary glands. We found that cell-autonomous disruption of ecdysone signaling through expression of the dominant negative $E c R^{F 645 A}$ in salivary glands blocked the loss of F-actin (Supplementary Fig. 3a). Conversely, addition of ecdysone to ex vivo cultures of salivary glands dissected at $-8 \mathrm{~h} \mathrm{PF}$ was sufficient to trigger F-actin breakdown, while addition of the translational inhibitor cycloheximide prevented ecdysone-triggered F-actin breakdown (Supplementary Fig. 3b), suggesting that translation of ecdysone-induced transcripts is critical for dismantling of cortical F-actin. Taken together, these results indicate that an ecdysone-induced sublethal pulse of $r p r$ triggers caspase activation at the cortex, which, in turn, initiates dismantling of the cortical F-actin cytoskeleton.

F-actin breakdown requires dronc localization and activity. Our data thus far suggests that $r p r$ activates caspases at the cortex, and it is this cortically restricted activity that breaks down the F-actin cytoskeleton. To understand the mechanisms that restrict caspase activity to the salivary gland cell cortex, we first examined the subcellular localization of Dronc protein. Staining with an 
a

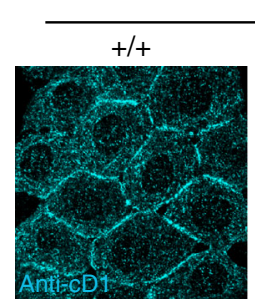

$+13.5 \mathrm{~h} \mathrm{PF}$

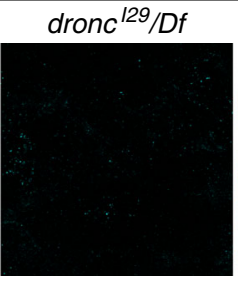

C

+12 h PF

$+/+$

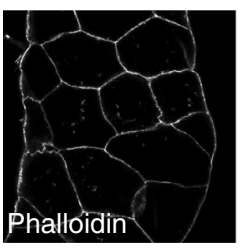

+13.5 h PF $+/+$

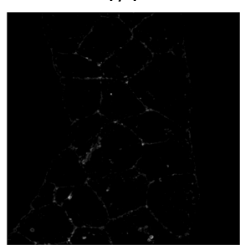
+ tango $i$

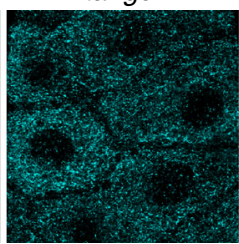

+ dark $i$

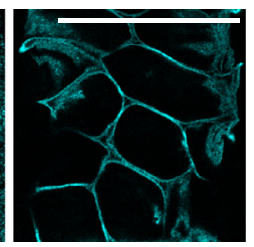

b

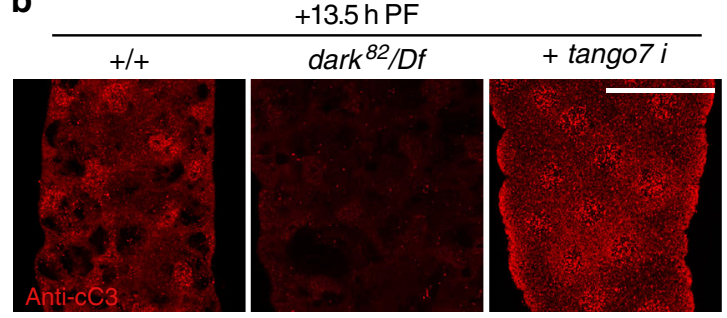

d

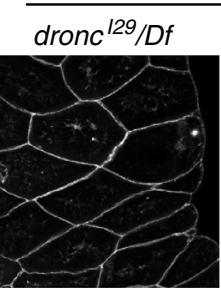

$+13.5 \mathrm{~h}$ PF

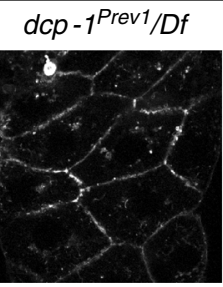

\section{drice $^{\Delta 1} / D f$}

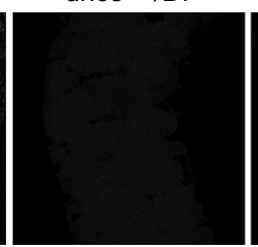

$\operatorname{dark}^{82} / D f$

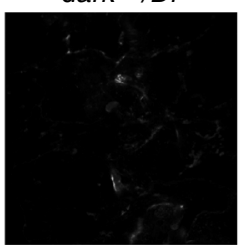

+ tango $i$

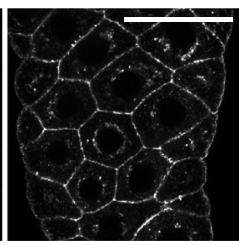

Fig. 4 Subcellular domains of caspase activation are independently regulated in dying salivary glands. a tango7 and dark regulate mutually exclusive subcellular domains of caspase activation in dying glands. Anti-cD1 staining, in cyan, is visible at both the cortex and in the cytoplasm of control salivary glands at $+13.5 \mathrm{~h} \mathrm{PF}$; both cortical and cytoplasmic staining is lost in dronc mutant salivary glands. Salivary gland-specific knockdown of tango 7 (with Sgs3-GAL4) does not affect cytoplasmic anti-cD1, but disrupts cortical anti-cD1. In contrast, salivary gland-specific knockdown of dark (with fkh-GAL4) disrupts cytoplasmic anti-cD1 but not cortical anti-cD1. b Cleaved caspase-3 (anti-cC3, in red), a diagnostic marker of caspase activation and apoptosis, does not require tango7. Anti-cC3 staining is visible only in the cytoplasm of control glands at $+13.5 \mathrm{~h}$ PF; this staining is lost in dark mutant animals. However, anti-cC3 staining is unaffected upon salivary gland-specific RNAi knockdown of tango7 (using Sgs3-GAL4). c Cortical F-actin is dismantled in dying salivary glands. The cortical F-actin cytoskeleton is intact in control salivary glands at $+12 \mathrm{~h}$ PF, just prior to the death response (phalloidin, in white). However, phalloidin staining shows that F-actin is dismantled at $+13.5 \mathrm{~h} \mathrm{PF}$. d F-actin dismantling during the death response requires dronc, $d c p-1$, and tango7. Phalloidin staining, in white, indicates that F-actin breakdown is disrupted in dronc and dcp-1 mutant and tango7-RNAi (Sgs3-GAL4) salivary glands at $+13.5 \mathrm{~h} \mathrm{PF}$, but F-actin breakdown is unaffected in drice and dark mutant salivary glands. Scale bars represent $100 \mu \mathrm{m}$. Df, deficiency, PF, puparium formation

anti-Dronc antibody showed that Dronc protein is enriched at the cortex at -8 and $-4 \mathrm{~h}$ PF (Supplementary Fig. 4a). Accordingly, overexpression of full-length Dronc showed preferential targeting to the cortex at -8 and $-4 \mathrm{~h} \mathrm{PF}$, but this cortical localization disappeared at PF (Fig. 3a; Supplementary Fig. 4b). Salivary gland-specific expression of dronc-RNAi abolished all Dronc staining at $-8 \mathrm{~h} \mathrm{PF}$ (Fig. 3a), confirming both the specificity of the antibody and efficiency of RNAi knockdown. Dronc protein localized to the cortex before induction of the rpr pulse that triggers cortical cleaved-Dcp-1 activation, suggesting that subcellular targeting of the initiator caspase may be responsible for restricting the rpr-initiated caspase cascade to the cell cortex. We next wanted to test the role of Dronc activity in F-actin remodeling, as caspases have previously been shown to regulate other non-apoptotic functions independently of their catalytic activity $^{30,31}$. Overexpression of Dronc accelerated the kinetics of F-actin breakdown in response to the small rpr pulse; in contrast, overexpression of a catalytically inactive dronc $\left(\right.$ dronc $\left.^{C-A}\right)$ prevented the breakdown of cortical F-actin (Fig. 3b). dronc $c^{C-A}$ still localized appropriately (Fig. 3b), suggesting that it may inhibit F-actin breakdown by displacing endogenous Dronc from the cortex. Taken together, these results demonstrate that both cortical localization and activity of Dronc are essential for F-actin dismantling during salivary gland development.

tango7 not dark regulates dronc localization and activity. We next wanted to determine how Dronc is localized to the cell cortex. Given that initiator caspases require adaptor proteins for activation and function $3,5,32$, we tested if the Apaf-1 adaptor protein dark, which is the canonical regulator of dronc activity in Drosophila ${ }^{33,34}$, was required for localization and/or function of dronc at the cortex. To our surprise, knockdown of dark had no effect on the cortical localization of Dronc protein (Fig. 3c). Moreover, dark null mutant glands still showed cortical anti-cD1 staining, and dismantling of cortical F-actin occurred normally (Fig. 3e). These results demonstrate that dark is not required for the localization or function of dronc at the salivary gland cell cortex. To identify other potential adaptor proteins for dronc in salivary glands, we conducted an RNAi-based screen among candidate proteins known to physically interact with dronc ${ }^{35-38}$. The strongest suppressor of F-actin breakdown in our screen was tango7 (Supplementary Fig. 5a). Salivary gland-specific knockdown of tango7 also disrupted cortical Dcp-1 activation (Supplementary Fig. 5b, c), indicating that tango7 plays a cell-autonomous role in caspase-dependent F-actin dismantling. tango 7 mutant glands also disrupted both the cortical activation of Dcp-1 and the dismantling of cortical F-actin (Fig. 3f). Consistent with a function in F-actin breakdown, we found that Tango7 protein localized to the salivary gland cell cortex. Analysis of Tango7 localization using recently generated endogenous superfolder GFP (sGFP)-tagged tango $7^{39}$ showed that Tango7 protein displayed subcellular dynamics similar to Dronc, with robust cortical localization at $-8 \mathrm{~h} \mathrm{PF}$ that was lost at $0 \mathrm{~h} \mathrm{PF}$ (Supplementary Fig. 5d, e). This places Tango7 protein at the right time and place to function with Dronc in F-actin dismantling. We also investigated the effect of tango 7 knockdown on Dronc protein stability, as previous studies have shown that loss of tango7 reduces Dronc protein levels ${ }^{37}$. However, western blot analysis of Dronc protein levels showed that knockdown of tango7 did not reduce Dronc expression in salivary glands (Fig. 3d). Importantly, tango7 appeared to be critical for proper cortical localization of Dronc. In tango7 knockdown glands, Dronc protein no longer displayed tight cortical localization and appeared instead to diffuse towards the cytoplasm, with 

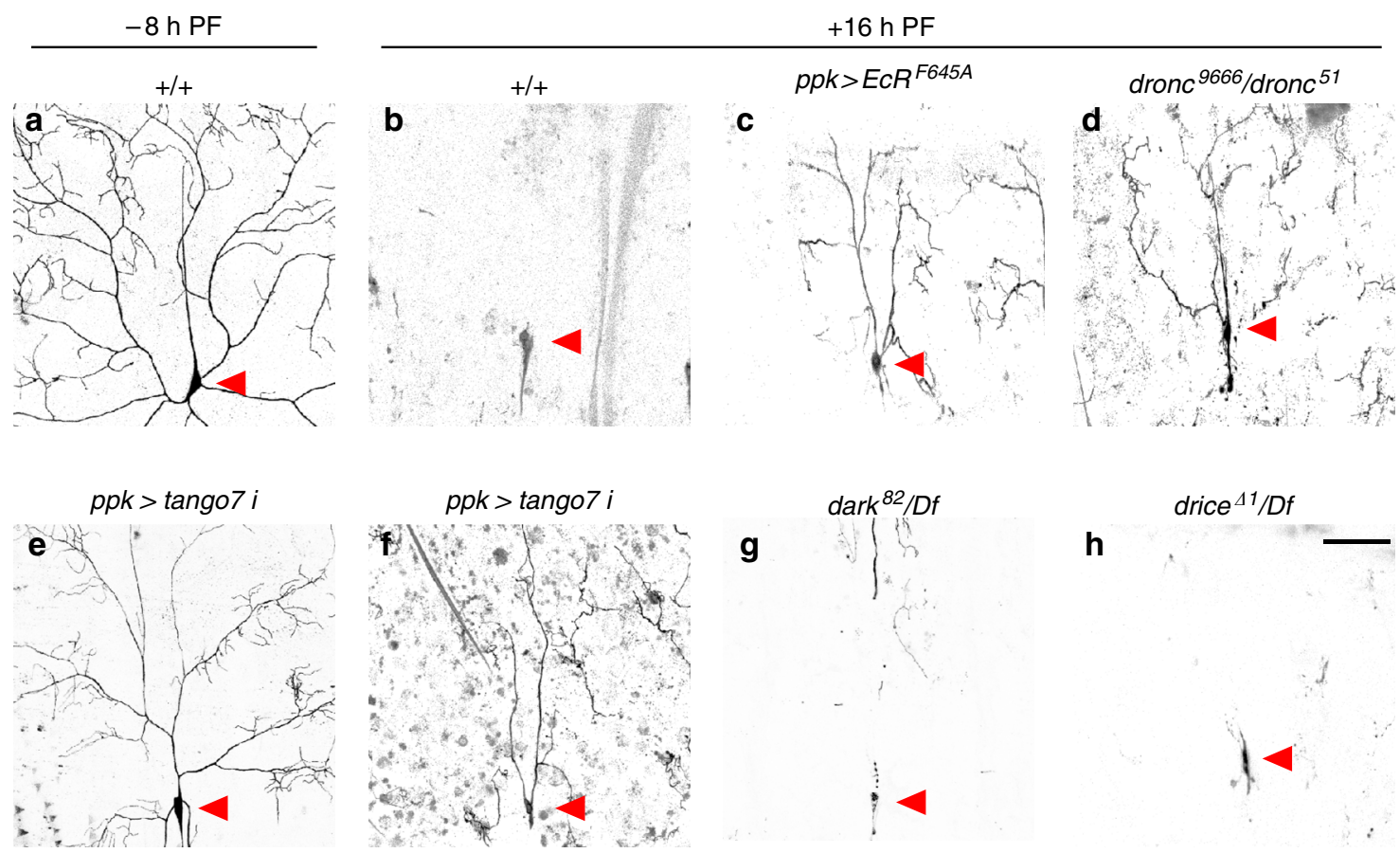

Fig. 5 Caspase-dependent pruning of class IV ddaC dendrites during metamorphosis requires tango 7 but not dark. a, b Dendritic arborizations, visualized by ppk-GAL4-driven expression of GFP, are present at $-8 \mathrm{~h} \mathrm{PF} \mathbf{a}$, but are completely pruned by $+16 \mathrm{~h}$ PF $\mathbf{b}$. $\mathbf{c}$, $\mathbf{d}$ Expression of a dominant-negative ecdysone receptor $\left(E c R^{F 645 A}\right)$ using ppk-GAL4 (c) or mutation of dronc (d) disrupts pruning at $+16 \mathrm{~h} \mathrm{PF}$. e, $\mathbf{f}$ Fewer dendritic arborizations form at $-8 \mathrm{~h} P F$ upon $\mathrm{RNAi}$ knockdown of tango7 (using ppk-GAL4) $\mathbf{e}$; however, these branches are not properly pruned at $+16 \mathrm{~h} \mathrm{PF} \mathrm{f.} \mathbf{g}, \mathbf{h}$ Dendritic pruning occurs normally in dark (g) or drice (h) mutant animals. Red arrowheads indicate the soma of ddaC neurons. Scale bars represent $100 \mu \mathrm{m}$. Df, deficiency, PF, puparium formation

conspicuous gaps lacking Dronc protein at the cortex (Fig. 3c). Taken together, these results demonstrate that tango 7 is required for localization, activation, and function of dronc in F-actin dismantling at the salivary gland cell cortex.

Caspase activation in mutually exclusive subcellular domains. Both dronc and dark play a critical role in caspase activation during the death response in salivary glands ${ }^{40-42}$, about half a day after the events discussed so far. To further examine the roles of tango7 and dark in the activation of dronc, we tested the role of these adaptors during the death response in salivary glands. At this later stage, anti-cD1 staining still showed robust signal at the cortex, but now also showed significant signal in the cytoplasm (Fig. 4a). Analysis of a timecourse of anti-cD1 staining showed that cortical activation is short-lived at this stage, while the cytoplasmic activation persisted longer (Supplementary Fig. 6), suggesting that cortical activation may be a transient event in response to the high-magnitude induction of $r p r$. Loss of dronc disrupted all anti-cD1 staining (Fig. 4a), indicating that Dcp-1 activation in both the cortical and cytoplasmic subcellular domains requires dronc activity. Strikingly, salivary gland-specific knockdown of tango7 disrupted anti-cD1 staining at the cortex without affecting anti-cD1 staining in the cytoplasm (Fig. 4a). Conversely, RNAi knockdown of dark disrupted anti-cD1 staining in the cytoplasm but not at the cortex (Fig. 4a). Consistent with previously published results, dark was required for anti-cC3 staining at this stage, which appeared to be only cytoplasmic (Fig. 4b). In contrast, knockdown of tango7 did not appear to affect cytoplasmic anti-cC3 staining (Fig. 4b), although tango7 knockdown appeared to disturb normal morphological breakdown during the death response. Anti-cC3 staining has previously been reported to detect dronc activity ${ }^{43}$, but our results suggest that this antibody specifically detects dark-dependent dronc activity in Drosophila tissues. Taken together, these results demonstrate that tango 7 is only required for caspase activation at the cortex, while dark is required for caspase activation in the cytoplasm. Importantly, activation of caspases in one subcellular domain does not appear to affect activation of caspases in the other domain, suggesting that caspase activation within these subcellular domains is restricted and compartmentalized.

We next tested if subcellular domain-specific activation of caspases resulted in compartment-specific functions. Cortical F-actin, which is transiently dismantled after the small pulse of $r p r$ at $\mathrm{PF}$, was dismantled once again during the death response (Fig. 4c). Similar to F-actin dismantling at PF, this second dismantling of cortical $\mathrm{F}$-actin in dying glands also required dronc and dcp-1, but not drice (Fig. 4d), demonstrating that a similar caspase cascade is responsible for the cortical function of caspases in both living and dying cells. Importantly, knockdown of tango 7 blocked breakdown of cortical F-actin in dying glands, despite the robust activation of caspases in the cytoplasm (Fig. 4a, b, d). Conversely, although loss of dark blocked activation of caspases in the cytoplasm (Fig. 4b), absence of dark did not disrupt dronc-dependent dismantling of cortical F-actin (Fig. 4d). Thus, contrary to commonly held assumptions, cytoplasmic activation of caspases does not result in widespread cellular caspase activity; instead, tango7 and dark appear to define mutually exclusive subcellular domains of dronc activation and function in dying salivary glands.

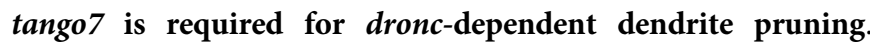
To determine the extent to which tango7-dependent control of dronc activity is used in other physiological contexts, we examined the role of tango7 in dronc-dependent pruning of dendritic arborizations during metamorphosis. Dendrites of the class IV ddaC sensory neurons are pruned in an ecdysone-dependent manner, and this pruning involves remodeling of the cortical F-actin cytoskeleton ${ }^{16,44-46}$. We found, 
a

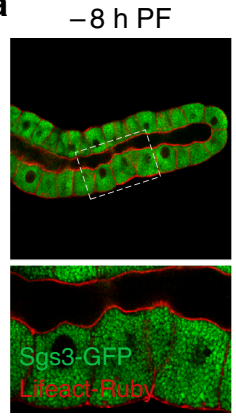

C

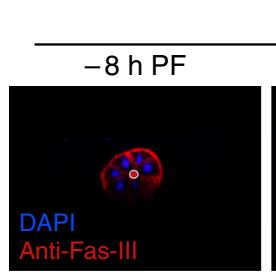

d

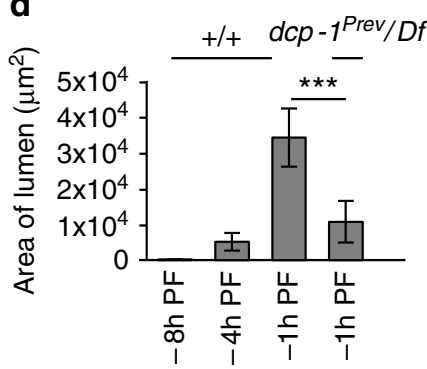

$-4 \mathrm{~h}$ PF

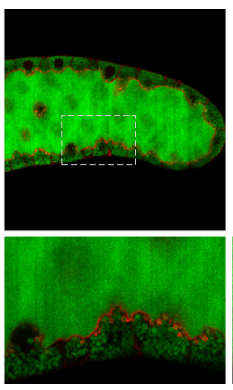

+ +

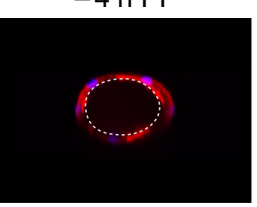

e

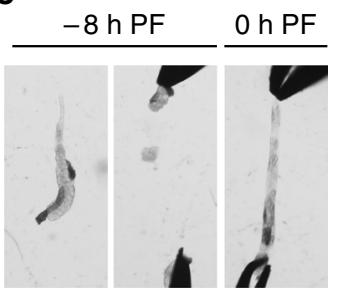

-1 h PF

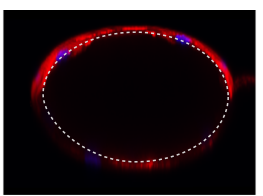

b $1 \mathrm{~h} P F$

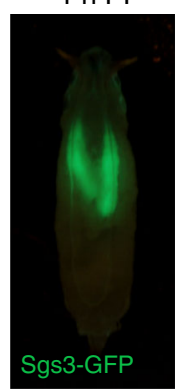

O h PF

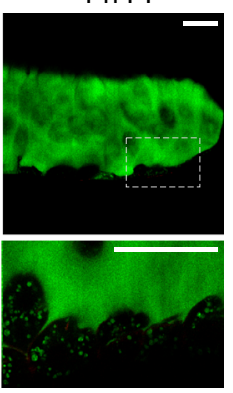

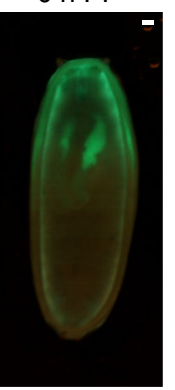

$d c p-1^{P r e v-1} / D f$

$-1 \mathrm{~h}$ PF

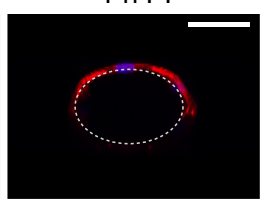

f

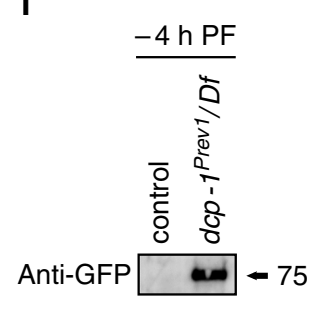

Fig. 6 Cortical caspase activation in living salivary glands controls tissue elasticity during expulsion of mucin-like glue proteins. a Dismantling of cortical F-actin in acinar cells coincides with secretion of glue proteins; boxed areas imaged at higher magnification below. At - $8 \mathrm{~h}$ PF, glue proteins (Sgs3-GFP, in green) are present only in the cells of the salivary gland. At $-4 \mathrm{~h} \mathrm{PF}$, exocytosis of glue proteins from cells into the lumen begins; by $-1 \mathrm{~h} P F$, all glue is present in the lumen. Lifeact-Ruby, in red, shows that $\mathrm{F}$-actin begins to break down at $-4 \mathrm{~h} \mathrm{PF}$, and dismantling is nearly complete by $-1 \mathrm{~h}$ PF. Salivary glands were imaged live/unfixed. $\mathbf{b}$ Glue proteins present in the lumen at $-1 \mathrm{~h} P F$ are expelled onto the surface of the animal at $0 \mathrm{~h} P F$. $\mathbf{c}$, $\mathbf{d}$ The lumen increases dramatically in size during glue exocytosis, and luminal expansion requires caspase activity. c Transverse view of salivary glands shows luminal expansion during glue secretion. Fasciclin-3 staining (anti-Fas-III, in red) marks septate junctions, nuclei stained with DAPI in blue. At $-8 \mathrm{~h}$ PF, salivary glands have a narrow lumen; however, as glue exocytosis progresses, the lumen expands dramatically until reaching maximal size at $-1 \mathrm{~h}$ PF. The lumen of $d c p-1$ mutant salivary glands does not expand normally. d Quantification of luminal area for stages and genotypes shown in c $(n=10$ per timepoint, error bars indicate s.d., asterisks indicate $p<0.01$ determined by one-tailed $t$-test). e Salivary gland elasticity is developmentally controlled. At $-8 \mathrm{~h} \mathrm{PF,} \mathrm{salivary} \mathrm{glands} \mathrm{are}$ rigid and tear at the slightest pull. In contrast, $\mathrm{O}$ h PF salivary glands can be stretched beyond their normal length. Equal force was applied to each stage; $n$ $=20$ tested per stage. $\mathbf{f}$ Luminal expansion is critical for timely expulsion of glue proteins. Glue expulsion normally occurs after larvae become stationary. Control wandering larvae at $-4 \mathrm{~h}$ PF do not expel glue, while $d c p-1$ mutant larvae precociously expel glue at this stage. Expelled glue proteins (in Sgs3-GFP animals) were collected and measured by western blot with anti-GFP antibodies. Scale bars represent $100 \mu \mathrm{m}$. Df, deficiency, PF, puparium formation

consistent with earlier reports ${ }^{16,17,44,45}$, that blocking ecdysone signaling or loss of dronc disrupted dendritic pruning (Fig. 5a-d); however, in contrast to a previous report ${ }^{16}$, we found that pruning occurred normally in dark mutant animals (Fig. 5g). Our result is consistent with axonal pruning in mammals, which requires caspase- 9 activity, but not Apaf- $1^{14}$. We also found that loss of drice did not block dendrite pruning (Fig. 5h), while knockdown of tango7 did (Fig. 5e, f). Although ddaC sensory neurons formed fewer dendritic branches upon knockdown of tango7, these branches were not properly pruned. These results demonstrate that the caspase cascade and regulatory mechanisms we defined in larval salivary glands also function during dendrite pruning, suggesting that the tango7-dependent pathway outlined here may represent a conserved and widely utilized mechanism for regulating cortical functions of caspases.

Caspase-dependent control of elasticity and timely secretion. In dying salivary glands, cortical F-actin is likely dismantled as a step toward cellular demolition; however, the function of F-actin breakdown in glands at the end of larval development was unclear. To understand why the cortical F-actin cytoskeleton is dismantled at the end of larval development, we first examined the most conspicuous function of glands at this stage: the secretion of mucin-like "glue" proteins. Glue secretion occurs in two discrete steps: first, exocytosis of glue-containing vesicles from acinar cells to the lumen (Fig. 6a); and second, expulsion of glue proteins from the lumen to the exterior, allowing the soon-to-be stationary pupa to adhere to a solid surface (Fig. 6b). Exocytosis of glue-containing vesicles has recently been shown to require the actin cytoskeleton ${ }^{47,}{ }^{48}$. However, imaging and western blot analysis showed that glue exocytosis occurred normally in dronc mutant glands (Supplementary Fig. 7), indicating that dronc-dependent breakdown of cortical F-actin is not required for this step of glue secretion.

Exocytosis of glue proteins results in a dramatic morphological change in the salivary glands. By $-1 \mathrm{~h} \mathrm{PF}$, glue exocytosis is nearly 
complete, but expulsion from the lumen onto the animal surface has not yet occurred. As a result, all of the secretory content is held within the lumen of the glands (Fig. 6a). Glue proteins are hygroscopic mucins which increase considerably in volume upon contact with water; therefore, the exocytosis and accumulation of secretory content resulted in a dramatic expansion of the lumen of the salivary glands (Fig. 6c, d). Luminal expansion began at $-4 \mathrm{~h}$ PF: the same time as F-actin breakdown, raising the question of whether there is a functional relationship between these two processes. We found that mutants of the ecdysone primary response gene $B R-C\left(r b p^{5}\right)$ produce very few gluecontaining vesicles; however, the vesicles that are produced are secreted normally. A reduced load of secretory content results in only slight luminal expansion in these glands, enabling a more detailed analysis of F-actin breakdown. Importantly, we found that F-actin is still dismantled in $r b p^{5}$ mutant salivary glands (Supplementary Fig. 8a), indicating that F-actin does not passively break as a result of luminal expansion. Additionally, high magnification analysis reveals clear "fraying" of the F-actin structure at $-1 \mathrm{~h} \mathrm{PF}$, causing F-actin to drop away from the cortex; this fraying is accompanied by complete dissolution of the F-actin meshwork (Supplementary Fig. 8b). Importantly, the fraying of F-actin is blocked in $r b p^{5}$ mutant salivary glands upon overexpression of diap1 or RNAi knockdown of rpr or tango7, further confirming that this process is caspase-dependent (Supplementary Fig. 8c), and suggesting that caspases may sever F-actin branchpoints during the breakdown process. Taken together, these results demonstrate that F-actin dismantling is an independently regulated process during salivary gland development.

We next tested if F-actin played a role in the second step of glue secretion: expulsion from the lumen onto the surface of the animal. Interestingly, the lumen of $d c p-1$ mutant or tango7 knockdown glands, which both failed to dismantle cortical F-actin, did not expand upon glue exocytosis (Fig. 5c, d; Supplementary Fig. 9), raising the possibility that F-actin dismantling facilitates "stretching" of the glands to accommodate increasing amounts of secretory content in the lumen. Consistently, at PF, when all cortical F-actin is gone, wild type salivary glands were extremely "elastic" and could easily be stretched well beyond their normal length (Fig. 5e). In contrast, glands at $-8 \mathrm{~h} \mathrm{PF}$, with an intact cortical F-actin cytoskeleton, tore when pulled (Fig. 5e). Finally, $d c p-1$ mutant salivary glands precociously expelled their secretory content prior to the onset of metamorphosis (Fig. 5f), indicating that the retained rigidity of the F-actin cytoskeleton in these animals did not permit luminal expansion to accommodate the large volume of secreted glue proteins. These results highlight a novel aspect of exocrine biology, in which caspase-dependent remodeling of cortical F-actin in acinar cells regulates tissue elasticity and facilitates storage of luminal content prior to its timely expulsion.

\section{Discussion}

Principles that govern the activation and function of caspases have fallen short in providing an understanding for how these enzymes can be activated to perform both delicate intracellular remodeling in living cells and total destruction in dying cells. In this paper, we provide new insights into the mechanisms that regulate caspase activation by comparing two completely different biological outcomes in the same tissue that both require caspase function. We show that the Drosophila homolog of caspase-9, dronc, is required for dismantling of the cortical F-actin cytoskeleton during salivary gland development-a role that is distinct from its known function in the salivary gland death response during metamorphosis. By systematically dissecting the regulation of dronc function at the cortex, we show that cortical functions of dronc are regulated independently from its cytoplasmic functions. The cytoplasmic functions of activated dronc require the canonical adaptor protein dark, while the cortical roles of dronc require tango7. In this manner, tango7 and dark restrict the function of dronc to distinct subcellular domains. Moreover, we also show that these two functions can be initiated independently through differential amplification of IAP antagonist expression, providing a model for how lethal and vital roles of caspases can be differentially activated in the same cell. Finally, we identify a new non-apoptotic function for caspases in the control of tissue elasticity to accommodate buildup of secreted products in the lumen of secretory tissues, facilitating their timely release.

Our results demonstrate that caspases can be activated in distinct, mutually exclusive subcellular domains within a single cell, and that these subcellular domains are generated by use of unique caspase adaptor proteins. Local activation of caspases, as detected by staining with antibodies to activated caspases, has been reported before; ${ }^{16-18,20,} 49$ however, here we demonstrate that local activation is achieved by targeting caspases to subcellular domains, and this targeting is necessary for subcellular functions of these caspases. Importantly, we show that caspases can be activated specifically in one domain without being activated in another, providing a mechanism that allows control of caspase activity with a previously unknown level of subcellular precision. However, the mechanisms that restrict caspase cascades to distinct subcellular compartments remain unclear. It is possible that caspase expression levels are intentionally kept low during non-lethal responses, and localized enrichment mediates subcellular domain-specific activation. For example, if most of the Dronc protein present in the cell localizes to the cortex, then this specific localization may restrict caspase functions to the cortical compartment. This model fits with our results at the end of larval development; however, in dying glands, caspases are independently activated in cortical and cytoplasmic compartments, suggesting that additional mechanisms are in play to restrict caspase activity to the appropriate subcellular compartment. For example, it is possible that caspase cascades occur within a physical complex consisting of initiator caspases, their adaptor proteins, effector caspases, and their substrates. In this model, only one of these proteins, likely the initiator caspase, would need to be subcellularly localized in order to generate a compartment-specific caspase cascade. However, resolution of this possible mechanism will require further studies. This subcellular domain-specific model for caspase activation contrasts with the commonly held belief that activated caspase cascades passively perpetuate themselves and spread throughout the cell, and also opens the possibility that caspases, through specific subcellular localization mediated by adaptor proteins, may play a role in many yet-to-be-identified biological processes.

We demonstrate that differential amplification of IAP antagonists at specific developmental stages determines lethal vs. non-lethal outcomes of caspase activation. In our system, differential amplification is accomplished through the use of transcription factors that function downstream of a steroid hormone signal. However, caspases must have an ability to "sense" the magnitude of the IAP antagonist pulse, ensuring that they initiate the appropriate lethal or non-lethal responses. One possible "sensing" mechanism may involve the aforementioned selectivity of initiator caspase adaptor proteins, like we observed with tango7 and dark. In this model, some adaptor protein complexes would require a lower IAP antagonist threshold for initiator caspase activation than others. However, elucidation of the detailed molecular mechanisms mediating 
"sensing" of IAP antagonist expression levels will require further study. Finally, our results indicate that small pulses of IAP antagonist expression are tissue specific, raising the possibility that many more of these pulses are generated in other tissues and developmental stages that have not yet been detected or characterized. Our data suggests that non-lethal, physiological functions of caspases may be more widespread than previously thought.

Our results show that caspases play a novel role during the secretion of glue proteins. Glue proteins are essential to allow a newly formed prepupa to adhere to a solid surface; however, when cortical F-actin dismantling fails, glue precociously "leaks" onto the surface of the animal. Although precocious expulsion of glue does not appear to have a deleterious effect in the lab, in the wild, it may adversely affect fitness by inhibiting larval movement or reducing the ability of the animal to stick securely to a surface during metamorphosis. Additionally, our results raise the question of whether other exocrine tissues in different species, such as the mammary gland, may utilize caspases in a similar manner to accommodate large amounts of secreted luminal products prior to their release.

In conclusion, systematic analysis of vital and lethal responses to caspase activation in the same cells has revealed mechanisms that allow caspases to be activated without killing the cell. Our results demonstrate that caspases can be activated in mutually exclusive subcellular domains, where activation of caspases in one domain does not trigger activation of caspases in another domain. We show that these subcellular domains are generated by different caspase adaptor proteins. It is likely that yet-to-be-identified adaptor proteins define other subcellular domains and, in so doing, help regulate the many physiological functions of caspases. Moreover, our results demonstrate that some of these subcellular domains have lower thresholds for activation of caspases, thereby allowing sublethal pulses of IAP antagonists to selectively initiate physiological functions of caspases. Together, these results outline a simple conceptual framework for controlling caspase activation during normal development and physiology.

\begin{abstract}
Methods
Fly strains and genetics. The following strains were obtained from the Bloomington Drosophila Stock Center: Sgs3-GFP, Dronc ${ }^{51}$, UAS-Lifeact-Ruby, Sgs3-Gal4, UAS-tango7-RNAi, E74A ${ }^{\text {neo24 }}$, br $^{\text {rpb5 }}$, UAS-EcR ${ }^{\text {F645A }}$, UAS-CD8-GFP, fkh-Gal4, ppk-Gal4, Df(3L)Exel6112 (for med24), Df(3L)81k19 (for E74A), Df(3R) BSC547 (for drice), Df(2R)BSC359 (for dark), Df(2R)BSC785 (for dcp-1), Df(3L) BSC282 (for dronc) and Df(3L)H99 (for rpr). The Vienna Drosophila RNAi Center provided Tango7-sGFP and the following RNAi lines: UAS-dronc-RNAi,

UAS-dark-RNAi, UAS-rpr-RNAi. Colleagues in the fly community provided the following stocks: dronc ${ }^{124}$ and dronc ${ }^{12950}$, drice ${ }^{\Delta 151}$, dark $^{8242}, \mathrm{dcp}^{\mathrm{P}} \mathrm{P}^{\text {Prev152 }}$ reaper $^{8753}$, UAS-dronc ${ }^{\mathrm{C}-\mathrm{A} 54}$, tango $^{\mathrm{E}}$ and tango $7^{\mathrm{L} 38^{\prime}}$, med24 ${ }^{\mathrm{pss5526}}$, UAS-dronc$\mathrm{GFP}^{34}$. All crosses were performed at $25^{\circ} \mathrm{C}$ in temperature-controlled incubators. Salivary gland-specific overexpression or RNAi knockdown was performed with the Sgs3-Gal4 driver unless otherwise noted. Wherever appropriate, control genotypes included an additional UAS transgene (UAS-GFP) to facilitate comparisons with experimental genotypes.
\end{abstract}

Developmental staging. All animals were raised at $25^{\circ} \mathrm{C}$ on cornmeal molasses media with granulated yeast. For staging third instar larvae, we combined the standard "blue food" technique ${ }^{55}$ with use of the mid-third instar transitionspecific reporter, Sgs3-GFP ${ }^{56}$. For $-8 \mathrm{~h}$ PF larvae, animals with "light blue" guts without glue secretion were collected. For $-4 \mathrm{~h}$ PF, animals with "clear blue" guts and glue in the lumen were selected. Finally, for $-1 \mathrm{~h}$ PF, we collected "clear" gut animals that were stationary with everted spiracles and soft cuticles. These staging methods have been independently tested for timing to PF. For animals after PF, animals were collected at $\mathrm{PF}$ and aged for the appropriate time at $25^{\circ} \mathrm{C}$ on a dampened filter paper plate. For stages at $12 \mathrm{~h}$ PF or later, we collected animals that were synchronized at head eversion (equivalent to $12 \mathrm{~h} P F$ ) and aged for the appropriate length of time thereafter.

Quantitative RT-PCR. mRNA expression levels of target genes were measured using quantitative real time PCR (qPCR). RNA was isolated from tissues dissected from appropriately staged animals using the RNeasy Plus Mini Kit (Qiagen). cDNA was synthesized from $400 \mathrm{ng}$ of total RNA using the SuperScript III First-Strand Synthesis System (Invitrogen). qPCR was performed on a Roche 480 LightCycler using LightCycler 480 SYBR Green I Master Mix (Roche). The target gene primer sequences used in this study were previously validated: $r p r^{57}, h i d^{57}, E 74 A^{58}$, and $r p 49^{59}$. In all cases, samples were run simultaneously with three independent biological replicates for each target gene. $r p 49$ was used as the reference gene. To calculate changes in relative expression and error bars, we used the Relative Expression Software Tool (REST) ${ }^{60}$. REST calculates confidence intervals and $p$-values for relative expression values using integrated bootstrapping and randomization methods. Standard error is calculated based on a confidence interval centered on the median; therefore error bars calculated by REST reflect asymmetrical tendencies in the data.

Immunofluorescent staining and image acquisition. Salivary glands dissected from appropriately staged animals were fixed for $30 \mathrm{~min}$ in PBS with $0.1 \%$ Triton X-100 (PBST) and $4 \%$ formaldehyde, blocked overnight with PBST/4\% BSA, and stained with the appropriate primary and secondary antibodies diluted in PBST $/ 4 \%$ BSA. All tissues were fixed and stained using identical conditions, and a wild type control was always done as a base line to detect any antibody signal loss. Identical microscope acquisition settings and image processing parameters were used for each experiment/set of paired samples. All primary antibodies were tested for consistency to reduce variability between lots, and the same lot was used for all experiments whenever possible. Primary antibodies used were: rabbit $\alpha$-Cleaved Dcp-1 (1:200; Cell Signaling \#9578, Lot 1), rabbit $\alpha$-Dronc (1:200; gift from P. Friesen, University of Wisconsin-Madison), rabbit $\alpha$-Cleaved Caspase-3 (1:200; Cell Signaling \#9661), and mouse anti-Fasciclin-3 (1:50; Developmental Studies Hybridoma Bank 7G10). Secondary antibodies used were AlexaFluor 488 anti-mouse and anti-rabbit (1:200; Invitrogen A11029 and A11034) and anti-rabbit Cy3 (1:200; Jackson Immuno-Research Labs 715-165-150). For DNA staining, DAPI was used (1:1000; Invitrogen). For phalloidin staining, salivary glands were dissected in PBS, fixed with $4 \%$ formaldehyde in PBS for $30 \mathrm{~min}$ and stained in the dark with a $100 \mathrm{nM}$ working solution of 488-conjugated Phalloidin (Life Technologies) for $30 \mathrm{~min}$. Stained tissues were mounted in Vectashield (Vector Laboratories). All images were captured on an Olympus FluoView FV1000 confocal microscope and optimized with FV10-ASW software. Images shown in figures are representative of at least three independent experiments with $n \geq 10$ tissues analyzed per experiment. Whenever possible, results were confirmed by an independent lab member who was blinded to the genotype/condition being analyzed. For 3D volume views, stained salivary glands were mounted in a $35 \mathrm{~mm}$ glass-bottom dish (MatTek Corporation) in $50^{\circ} \mathrm{C} 0.8 \%$ agarose and imaged immediately using a $Z$-stack sequence at $3 \mu \mathrm{m}$ intervals on a Nikon A1R confocal microscope. Volume views were optimized using the Nikon NIS Element software, and lumen size quantification was done using ImageJ. Light microscope images of whole salivary glands were taken on an Olympus SZX16 stereomicroscope using an Olympus DP72 digital camera with DP2-BSW software.

Ex vivo salivary gland cultures. Ex vivo cultures were performed using standard methods ${ }^{61}$. Salivary glands were dissected in oxygenated Schneider's insect media (Sigma) and pre-incubated for $30 \mathrm{~min}$ in a "lung" with flowing oxygen. Fresh oxygenated media with $5 \mu \mathrm{M}$ of 20 -hydroxyecdysone (Sigma) and/or $85 \mu \mathrm{M}$ cycloheximide (Sigma) was added to the appropriate samples. Salivary glands were stained and imaged as described above.

Glue secretion assays. For glue protein imaging, salivary glands from strains carrying the Sgs3-GFP fusion protein were dissected in PBS and immediately imaged live on an Olympus FluoView FV1000 confocal microscope. For glue secretion western blot assays, three appropriately staged animals carrying Sgs3-GFP were collected, washed with water, and the glue content that had not been expelled was measured. The animals were homogenized in $50 \mu \mathrm{l}$ of hi-salt lysis buffer (25 mM HEPES pH 7.4, $300 \mathrm{mM} \mathrm{NaCl}, 1.5 \mathrm{mM} \mathrm{MgCl}, 1 \mathrm{mM}$ EDTA, 0.5\% Triton X-100, $50 \mathrm{mM} \beta$-glycero-phosphate, $50 \mathrm{mM} \mathrm{NaF}, 1 \mathrm{mM} \mathrm{Na}_{3} \mathrm{V0}_{4}, 5 \mathrm{mg} / \mathrm{ml}$ Pepstatin A, 1 mM DTT, $5 \mathrm{mg} / \mathrm{ml}$ Aprotinin, $200 \mathrm{mM}$ PMSF, $10 \mathrm{mg} / \mathrm{ml}$ Leupeptin, $4 \mathrm{nM}$ Microsystin). In total, $10 \mu \mathrm{l}$ of each sample was then subjected to a western blot analysis. To monitor premature expulsion, five wandering larvae (at $-8 \mathrm{~h} \mathrm{PF}$ ) of the appropriate genotype were placed in small PCR tubes, allowed to wander for $2 \mathrm{~h}$, and then removed. Only samples lacking animals that had started PF were used; $20 \mu \mathrm{l} 2 \times$ sample buffer (1.0 M Tris-HCl pH 6.8, $8 \%$ SDS, $40 \%$ Glycerol, $20 \%$ 2-Mercaptoethanol) was added to the small PCR tubes and vortexed vigorously to collect expelled proteins. The samples were heated at $95^{\circ} \mathrm{C}$ for $5 \mathrm{~min}$ and were subjected to a western blot analysis.

Western blot analysis. Protein expression levels were measured by western blot analysis. Primary antibodies used were rabbit anti-GFP (1:2000; Torrey Pines TP401), rabbit anti-Dronc (1:1000; gift from P. Friesen, University of Wisc onsin-Madison), mouse anti- $\beta$-tubulin (1:1000; Millipore \#05-661), and rabbit anti- $\beta$-actin (1:1000; Cell Signaling \#4967). Secondary antibodies used were alkaline phosphatase-conjugated goat anti-rabbit and anti-mouse IgG (1:30,000; Sigma A3687 and A3438). Membranes were developed for imaging with ECF 
substrate (GE Healthcare) and were imaged using a Storm 840 Scanner (Amersham Bioscience) and processed with ImageQuant TL software version 7.0 (GE Healthcare). Full-length, uncropped blots are included in Supplementary Fig. 10.

Dendrite pruning. Dendritic arborizations of the ddaC neurons on the larval abdomen were imaged by expression of CD8-GFP driven by $p p k-G a l 4^{46}$. For $-8 \mathrm{~h}$ $\mathrm{PF}$, the larvae were placed in a drop of PBS and pushed between a coverslip and microscope slide until the animals were immobile but still alive. For $16 \mathrm{~h} \mathrm{PF}$, the cuticle of appropriately staged pupae was carefully removed and the animals were mounted in PBS. All mounted samples were imaged immediately on an Olympus FluoView FV1000 confocal microscope with 10-25 optical sections at $1.5 \mu \mathrm{m}$ intervals. Images were optimized using the FV10-ASW software and inverted using Photoshop CC 2015.

Data availability. All relevant data is provided in the paper and supplemental supporting information.

Received: 15 August 2016 Accepted: 20 July 2017

Published online: 19 September 2017

\section{References}

1. Mcllwain, D. R., Berger, T. \& Mak, T. W. Caspase functions in cell death and disease. Cold Spring Harb. Perspect. Biol. 5, a008656-a008656 (2013).

2. Taylor, R. C., Cullen, S. P. \& Martin, S. J. Apoptosis: controlled demolition at the cellular level. Nat. Rev. Mol. Cell Biol. 9, 231-241 (2008).

3. Salvesen, G. S. \& Dixit, V. M. Caspase activation: the induced-proximity model. Proc. Natl Acad. Sci. USA 96, 10964-10967 (1999).

4. Riedl, S. J. \& Salvesen, G. S. The apoptosome: signalling platform of cell death. Nat. Rev. Mol. Cell Biol. 8, 405-413 (2007).

5. Bratton, S. B. \& Salvesen, G. S. Regulation of the Apaf-1-caspase-9 apoptosome. J. Cell Sci. 123, 3209-3214 (2010).

6. Stennicke, H. R. et al. Pro-caspase-3 is a major physiologic target of caspase-8. J. Biol. Chem. 273, 27084-27090 (1998).

7. Stennicke, H. R. \& Salvesen, G. S. Properties of the caspases. Biochim. Biophys. Acta 1387, 17-31 (1998).

8. Shalini, S., Dorstyn, L., Dawar, S. \& Kumar, S. Old, new and emerging functions of caspases. Cell Death Differ. 22, 526-539 (2015).

9. Yamaguchi, Y. \& Miura, M. Programmed cell death and caspase functions during neural development. Curr. Top. Dev. Biol. 114, 159-184 (2015).

10. Unsain, N. \& Barker, P. A. New views on the misconstrued: executioner caspases and their diverse non-apoptotic roles. Neuron 88, 461-474 (2015).

11. Nakajima, Y.-I. \& Kuranaga, E. Caspase-dependent non-apoptotic processes in development. Cell Death Differ. 24, 1422-1430 (2017).

12. Solier, S., Fontenay, M., Vainchenker, W., Droin, N. \& Solary, E. Non-apoptotic functions of caspases in myeloid cell differentiation. Cell Death Differ. 24, 1337-1347 (2017).

13. Fogarty, C. E. \& Bergmann, A. Killers creating new life: caspases drive apoptosis-induced proliferation in tissue repair and disease. Cell Death Differ. 24, 1390-1400 (2017).

14. Cusack, C. L., Swahari, V., Hampton Henley, W., Michael Ramsey, J. \& Deshmukh, M. Distinct pathways mediate axon degeneration during apoptosis and axon-specific pruning. Nat. Commun. 4, 1876 (2013).

15. Vesela, B. et al. Non-apoptotic role for caspase-7 in hair follicles and the surrounding tissue. J. Mol. Histol. 46, 443-455 (2015).

16. Williams, D. W., Kondo, S., Krzyzanowska, A., Hiromi, Y. \& Truman, J. W. Local caspase activity directs engulfment of dendrites during pruning. Nat. Neurosci. 9, 1234-1236 (2006)

17. Kuo, C. T., Zhu, S., Younger, S., Jan, L. Y. \& Jan, Y.-N. Identification of E2/E3 ubiquitinating enzymes and caspase activity regulating Drosophila sensory neuron dendrite pruning. Neuron 51, 283-290 (2006).

18. Arama, E., Agapite, J. \& Steller, H. Caspase activity and a specific cytochrome C are required for sperm differentiation in Drosophila. Dev. Cell 4, 687-697 (2003).

19. Arama, E., Bader, M., Srivastava, M., Bergmann, A. \& Steller, H. The two Drosophila cytochrome $\mathrm{C}$ proteins can function in both respiration and caspase activation. EMBO J. 25, 232-243 (2006).

20. Huh, J. R. et al. Multiple apoptotic caspase cascades are required in nonapoptotic roles for Drosophila spermatid individualization. PLoS Biol. 2, E15 (2004).

21. Vasudevan, D. \& Ryoo, H. D. Regulation of cell death by IAPs and their antagonists. Curr. Top. Dev. Biol. 114, 185-208 (2015).

22. Jiang, C., Baehrecke, E. H. \& Thummel, C. S. Steroid regulated programmed cell death during Drosophila metamorphosis. Development 124, 4673-4683 (1997).
23. Jiang, C., Lamblin, A. F., Steller, H. \& Thummel, C. S. A steroid-triggered transcriptional hierarchy controls salivary gland cell death during Drosophila metamorphosis. Mol. Cell 5, 445-455 (2000).

24. Warren, J. T. et al. Discrete pulses of molting hormone, 20-hydroxyecdysone during late larval development of Drosophila melanogaster: correlations with changes in gene activity. Dev. Dyn. 235, 315-326 (2006).

25. Lee, C. Y., Simon, C. R., Woodard, C. T. \& Baehrecke, E. H. Genetic mechanism for the stage- and tissue-specific regulation of steroid triggered programmed cell death in drosophila. Dev. Biol. 252, 138-148 (2002).

26. Ihry, R. J. \& Bashirullah, A. Genetic control of specificity to steroid-triggered responses in Drosophila. Genetics 196, 767-780 (2014).

27. Yin, V. P., Thummel, C. S. \& Bashirullah, A. Down-regulation of inhibitor of apoptosis levels provides competence for steroid-triggered cell death. J. Cell Biol. 178, 85-92 (2007).

28. Riedl, J. et al. Lifeact: a versatile marker to visualize F-actin. Nat. Methods $\mathbf{5}$, 605-607 (2008)

29. Melak, M., Plessner, M. \& Grosse, R. Actin visualization at a glance. J. Cell Sci. 130, 525-530 (2017).

30. Li, J. et al. Caspase-11 regulates cell migration by promoting Aip1-Cofilinmediated actin depolymerization. Nat. Cell Biol. 9, 276-286 (2007).

31. Kamber Kaya, H. E., Ditzel, M., Meier, P. \& Bergmann, A. An inhibitory monoubiquitylation of the Drosophila initiator caspase Dronc functions in both apoptotic and non-apoptotic pathways. PLoS Genet. 13, el006438 (2017).

32. Snipas, S. J., Drag, M., Stennicke, H. R. \& Salvesen, G. S. Activation mechanism and substrate specificity of the Drosophila initiator caspase DRONC. Cell Death Differ. 15, 938-945 (2008).

33. Rodriguez, A. et al. Dark is a Drosophila homologue of Apaf-1/CED-4 and functions in an evolutionarily conserved death pathway. Nat. Cell Biol. 1, 272-279 (1999)

34. Quinn, L. M. et al. An essential role for the caspase dronc in developmentally programmed cell death in Drosophila. J. Biol. Chem. 275, 40416-40424 (2000).

35. Gramates, L. S. et al. FlyBase at 25: looking to the future. Nucleic Acids Res. 45 , D663-D671 (2017)

36. Guruharsha, K. G. et al. A protein complex network of Drosophila melanogaster. Cell 147, 690-703 (2011).

37. Chew, S. K. et al. Genome-wide silencing in Drosophila captures conserved apoptotic effectors. Nature 460, 123-127 (2009).

38. D'Brot, A. et al. Tango7 directs cellular remodeling by the Drosophila apoptosome. Genes Dev. 27, 1650-1655 (2013).

39. Sarov, M. et al. A genome-wide resource for the analysis of protein localisation in Drosophila. eLife 5, e12068 (2016).

40. Daish, T. J., Mills, K. \& Kumar, S. Drosophila caspase DRONC is required for specific developmental cell death pathways and stress-induced apoptosis. Dev. Cell 7, 909-915 (2004).

41. Martin, D. N. \& Baehrecke, E. H. Caspases function in autophagic programmed cell death in Drosophila. Development 131, 275-284 (2004).

42. Akdemir, F. et al. Autophagy occurs upstream or parallel to the apoptosome during histolytic cell death. Development 133, 1457-1465 (2006).

43. Fan, Y. \& Bergmann, A. The cleaved-Caspase-3 antibody is a marker of Caspase-9-like DRONC activity in Drosophila. Cell Death Differ. 17, 534-539 (2010).

44. Williams, D. W. \& Truman, J. W. Cellular mechanisms of dendrite pruning in Drosophila: insights from in vivo time-lapse of remodeling dendritic arborizing sensory neurons. Development 132, 3631-3642 (2005).

45. Kuo, C. T., Jan, L. Y. \& Jan, Y.-N. Dendrite-specific remodeling of Drosophila sensory neurons requires matrix metalloproteases, ubiquitin-proteasome, and ecdysone signaling. Proc. Natl Acad. Sci. USA 102, 15230-15235 (2005).

46. Kirilly, D. et al. A genetic pathway composed of Sox14 and Mical governs severing of dendrites during pruning. Nat. Neurosci. 12, 1497-1505 (2009).

47. Rousso, T., Schejter, E. D. \& Shilo, B.-Z. Orchestrated content release from Drosophila glue-protein vesicles by a contractile actomyosin network. Nat. Cell Biol. 18, 181-190 (2016).

48. Tran, D. T., Masedunskas, A., Weigert, R. \& Ten Hagen, K. G. Arp2/3mediated F-actin formation controls regulated exocytosis in vivo. Nat. Commun. 6, 10098 (2015).

49. Campbell, D. S. \& Okamoto, H. Local caspase activation interacts with Slit-Robo signaling to restrict axonal arborization. J. Cell Biol. 203, 657-672 (2013).

50. Xu, D., Li, Y., Arcaro, M., Lackey, M. \& Bergmann, A. The CARD-carrying caspase Dronc is essential for most, but not all, developmental cell death in Drosophila. Development 132, 2125-2134 (2005).

51. Muro, I. et al. The Drosophila caspase Ice is important for many apoptotic cell deaths and for spermatid individualization, a nonapoptotic process. Development 133, 3305-3315 (2006).

52. Laundrie, B. et al. Germline cell death is inhibited by P-element insertions disrupting the dcp-1/pita nested gene pair in Drosophila. Genetics 165, 1881-1888 (2003). 
53. Moon, N.-S. et al. E2F and p53 induce apoptosis independently during Drosophila development but intersect in the context of DNA damage. PLoS Genet. 4, e1000153 (2008)

54. Meier, P., Silke, J., Leevers, S. J. \& Evan, G. I. The Drosophila caspase DRONC is regulated by DIAP1. EMBO J. 19, 598-611 (2000).

55. Andres, A. J. \& Thummel, C. S. Methods for quantitative analysis of transcription in larvae and prepupae. Methods Cell Biol. 44, 565-573 (1994).

56. Biyasheva, A., Do, T. V., Lu, Y., Vaskova, M. \& Andres, A. J. Glue secretion in the Drosophila salivary gland: a model for steroid-regulated exocytosis. Dev. Biol. 231, 234-251 (2001).

57. Ihry, R. J., Sapiro, A. L. \& Bashirullah, A. Translational control by the DEAD Box RNA helicase belle regulates ecdysone-triggered transcriptional cascades. PLoS Genet. 8, e1003085 (2012).

58. Caldwell, P. E., Walkiewicz, M. \& Stern, M. Ras activity in the Drosophila prothoracic gland regulates body size and developmental rate via ecdysone release. Curr. Biol. 15, 1785-1795 (2005).

59. Denton, D. et al. Autophagy, not apoptosis, is essential for midgut cell death in Drosophila. Curr. Biol. 19, 1741-1746 (2009).

60. Pfaffl, M. W., Horgan, G. W. \& Dempfle, L. Relative expression software tool (REST) for group-wise comparison and statistical analysis of relative expression results in real-time PCR. Nucleic Acids Res. 30, e36 (2002).

61. Bashirullah, A. et al. Coordinate regulation of small temporal RNAs at the onset of Drosophila metamorphosis. Dev. Biol. 259, 1-8 (2003).

\section{Acknowledgements}

Stocks obtained from the Bloomington Drosophila Stock Center (NIH P40OD018537) were used in this study. We are grateful to P. Friesen (Madison, WI, USA) and the DSHB (Iowa City, IA, USA) for antibodies and colleagues in the Drosophila community for fly stocks; Gina Castelvecchi for technical assistance; Khailee Marischuk for critical reading of the manuscript and helpful discussions. This work was supported in part by the National Science Foundation (GRFP DGE-1256259 to S.D.N.) and the National Institutes of Health (GM095944 to A.B.).

\section{Author contributions}

A.B. and Y.K. conceived and designed the project. Y.K. and S.D.N. performed the experiments and prepared the figures. Y.K., S.D.N., and A.B. analyzed the data and wrote the manuscript. A.B. supervised all aspects of the project.

\section{Additional information}

Supplementary Information accompanies this paper at doi:10.1038/s41467-017-00693-3.

Competing interests: The authors declare no competing financial interests.

Reprints and permission information is available online at http://npg.nature.com/ reprintsandpermissions/

Publisher's note: Springer Nature remains neutral with regard to jurisdictional claims in published maps and institutional affiliations.

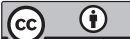

Open Access This article is licensed under a Creative Commons Attribution 4.0 International License, which permits use, sharing, adaptation, distribution and reproduction in any medium or format, as long as you give appropriate credit to the original author(s) and the source, provide a link to the Creative Commons license, and indicate if changes were made. The images or other third party material in this article are included in the article's Creative Commons license, unless indicated otherwise in a credit line to the material. If material is not included in the article's Creative Commons license and your intended use is not permitted by statutory regulation or exceeds the permitted use, you will need to obtain permission directly from the copyright holder. To view a copy of this license, visit http://creativecommons.org/ licenses/by/4.0/.

(C) The Author(s) 2017 